20

\title{
Исследование нейроваскулярных структур с помощью фазово-модуляционной спектрофотометрии*
}

\author{
(ㄱ Л.П. Сафоонова ${ }^{1}$, В.Г. Орлова ${ }^{1}$, А.Н. Шкарубо ${ }^{2}$ \\ ${ }^{1}$ Московский государственный технический университет им. Н.Э. Баумана, \\ 105005 Москва, Россия \\ ${ }^{2}$ Национальный медицинский исследовательский центр нейрохирургии им. академика Н.Н. Бурденко, \\ 125047 Москва, Россия \\ e-mail: larisa.safonova@gmail.com
}

Поступила в редакцию 08.01.2019 г.

В окончательной редакции 22.02.2019 г.

Принята к публикации 26.02.2019 г.

Исследована возможность применения фазово-модуляционной спектрофотометрии для обнаружения и распознавания крупных кровеносных сосудов и нервов в объеме биологических тканей в задачах нейрохирургии с эндоскопическим трансназальным доступом при удалении опухолей основания черепа. Изучены оптические и динамические характеристики, присущие различным типам нейроваскулярных структур. Предложены и экспериментально исследованы in vivo и in situ информативные независимые параметры и соответствующие им критерии обнаружения и распознавания нейроваскулярных структур в объеме ткани, основанные на различии оптических свойств крови, нервов и окружающих их тканей. Полученные предварительные результаты свидетельствуют о перспективности применения метода фазово-модуляционной спектрофотометрии в эндоскопической нейрохирургии и могут использоваться в спектрофотометрии с импульсным временным подходом.

DOI: $10.21883 /$ OS.2019.06.47778.58-19

\section{Введение}

Современные успехи в развитии малоинвазивных технологий особенно важны для нейрохирургии, которой присущи наибольшая сложность проведения манипуляций и слишком высокая цена за возможные ошибки [14]. Примером комплексного использования новых решений являются нейроонкология и активно развивающаяся эндоскопическая хирургия опухолей основания черепа с трансназальным доступом - наиболее сложная область нейрохирургии [1-3]. Основные проблемы связаны с необходимостью обеспечить максимально возможную радикальность удаления опухолевой ткани при минимизации постоперационных осложнений, а также с сохранением анатомической целостности и функциональности жизненно важных структур мозга, магистральных артерий и черепных нервов, особенно в условиях прорастания их опухолевой тканью и изменения анатомической топографии. В связи с этим актуальной является разработка методов и средств диагностики in situ для обнаружения и распознавания нейроваскулярных структур в объеме опухолевой ткани в процессе операции.

Основными требованиями к методу и устройству диагностики нейроваскулярных структур являются следующие:

\footnotetext{
* The 22nd Annual Conference Saratov Fall Meeting 2018 (SFM'18): VI International Symposium „Optics and Biophotonics“ and XXII International School for Junior Scientists and Students on Optics, Laser Physics \& Biophotonics, September 24-29, 2018, Saratov, Russia. https://www.sgu.ru/structure/fiz/saratov-fall-meeting/previousconferences/saratov-fall-meeting-2018
}

- малые габариты датчика и потенциальная возможность совмещения с эндоскопическим инструментом хирурга;

- совместимость с условиями проведения эндоскопической операции в малом объеме и при наличии засветки;

- возможность глубинного зондирования, обнаружения и распознавания магистральных артерий и черепных нервов в объеме ткани;

- возможность многократного применения в процессе выполнения операции по удалению опухоли;

- применимость для различных типов опухолей основания черепа;

- отсутствие дополнительных рисков для пациента и медицинского персонала от применения метода диагностики;

- экономичность метода.

При выполнении основных требований, специальные требования направлены на соответствие разрабатываемого метода и устройства идеальному решению, которое обеспечивает:

- возможность бесконтактного зондирования;

- совмещение с дополнительными функциями, позволяющими повысить качество, эффективность и безопасность нейрохирургического вмешательства трансназального доступа; к таким функциям относятся методы и технические решения для повышения достоверности распознавания черепных нервов, для повышения чувствительности метода к нейроваскулярным структурам 
малого диаметра, для обнаружения патологических клеток и тканей с высоким пространственным разрешением;

- потенциальную возможность применения в роботизированной нейрохирургии.

Количественные критерии по каждому из требований на текущем этапе разработок необходимо уточнять или разрабатывать ввиду относительной новизны решаемой задачи и отсутствия четких медицинских требований.

Для повышения радикальности удаления опухоли путем идентификации и обнаружения границ опухолевой ткани, для дифференцирования тканей и клеток, оценки их функциональной активности, оценки локальной гемодинамики и состояния капилляров, для обеспечения навигации и ориентирования в ране хорошо зарекомендовали себя оптические методы, такие как рамановская спектроскопия in vivo [5], широкополосная [6] и узкополосная спектроскопия [7], оптическая когерентная томография $[9,10]$, флуоресцентая спектроскопия и визуализация [11-13] и мультимодальные сочетанные решения [6]. Новым перспективным направлением для интраоперационной дифференциальной диагностики опухолевых клеток и тканей является дистанционная неразрушающая терагерцовая спектроскопия и визуализация $[14,15]$. Основными ограничениями указанных оптических методов являются малая глубина зондирования ткани, около $1.5 \mathrm{~mm}$, отсутствие стандартных методик применения в клинической практике или технических решений, применимых in situ для нейрохирургии трансназального доступа. Большинство технических решений направлено на совмещение средств диагностики с эндоскопическим инструментом. Конструктивные решения оптоволоконных датчиков для различных применений рассмотрены в [16].

Для обнаружения и распознавания магистральных артерий применяется метод ультразвуковой допплерографии со специальным эндоскопическим датчиком для нейрохирургии [17]. Существенными ограничениями являются отдельно вводимый трансназально инструмент и невозможность распознавания артерии в случае, если направление распространения ультразвуковой волны перпендикулярно линейной скорости кровотока в сосуде, что возможно в условиях малого операционного поля. Для идентификации двигательных черепных нервов в последние годы используется триггерная электромиография (т-ЭМГ, t-EMG), при которой регистрируется потенциал действия мышцы (compound muscle action potential), возникающий в ответ на стимуляцию нервного ствола электрическим током [3]. Нейрофизиологическая идентификация черепных нервов в несколько раз снижает частоту их поражения и сопутствующее снижение качества жизни пациентов [3]. Метод т-ЭМГ широко применяется в малоинвазивной хирургии позвоночника [18], характеризуется наличием стандартной методики, но имеет ряд ограничений [19]. Основными недостатками т-ЭМГ являются широкая зона распространения тока по электропроводящим тканям, что может привести к ложному ответу на стимуляцию, и необходимость регистрации отклика дополнительными измерительными средствами вне области локализации нерва. В последнее время ведутся исследования по оптической стимуляции нерва с помощью лазерных импульсов ближнего инфракрасного диапазона [19-24]. На сегодняшний день не существует стандартной методики нейростимуляции с применением энергии лазерного излучения. Тип источника, длина волны стимулирующего излучения, плотность энергии, длительность и частота следования импульсов, диаметр и апертурный угол пучка и метод регистрации наличия стимула зависят от области применения, объекта и целей исследования.

Ультразвуковая визуализация нервов и сосудов, представленная в [25], не предназначена для малого операционного поля эндоназальной нейрохирургии. Идентификация проводится путем анализа получаемых, трудно интерпретируемых, изображений. Качество современной нейрохирургии во многом обеспечивается на предоперационном этапе и интраоперационно сложными навигационными системами и комплексами на основе методов магнитно-резонансной томографии (МРТ), позитронноэмиссионной томографии (ПЭТ), рентгеновской компьютерной томографии (КТ), ультразвукового сканирования (УЗИ) и сочетаний методов (КТ/МРТ, ПЭТ/КТ, ПЭТ/МРТ и др.) [26,27]. Существенными ограничениями отдельных из указанных методов и технических реализаций являются сложность или невозможность обеспечения оперативной диагностики в малом операционном поле локально, высокая стоимость, высокий уровень лучевой нагрузки и дополнительные риски.

Метод спектрофотометрии биологических тканей в видимом и ближнем инфракрасном диапазонах длин волн, известный как NIRS (near infrared spectroscopy), активно развивается в последние три десятилетия и имеет широкую область применения в медицине и нейрофизиологии [28-33]. Для дифференциального анализа состава, структуры и функциональной активности биологических тканей наиболее перспективны реализации NIRS на основе режимов с временным разрешением (time resolved spectroscopy, TRS): 1) частотного (frequencydomain, FD) или, иначе, фазово-модуляционного (ФМ, phase modulated, PM) и 2) временного (time-domain, TD) или импульсного. На основе указанных подходов возможна количественная оценка, оперативный контроль и длительный мониторинг оптических параметров, коэффициента поглощения и транспортного коэффициента рассеяния, косвенная количественная оценка структурных особенностей и компонентного состава биологических тканей и ряда физиологических параметров, характеризующих локальную гемодинамику и метаболизм биоткани [28-33]. Теоретические основы TD- и PMподходов, особенности их технических реализаций и варианты применений подробно рассмотрены в литературе $[31,32]$.

Наиболее функциональным, перспективным, но сложным в технической реализации, на сегодняшний день 
является TD-подход (TD NIRS). Как следует из недавних научных публикаций, на основе данного подхода возможны бесконтактные измерения при малом или „нулевом“ расстоянии (одноволоконно) между источником и приемником, зондирование биологической ткани на глубину до $6 \mathrm{~cm}$ с высоким временным разрешением в расширенном спектральном диапазоне 500-1000 m и с количественной оценкой оптических параметров $[34,35]$. Однако данная методика в настоящий момент находится на стадии разработки.

Целью данной работы является экспериментальное исследование применимости ФМ спектрофотометрии (ФМС) для обнаружения и распознавания крупных кровеносных сосудов и нервов в объеме биологической ткани. Для достижения цели решались следующие задачи: анализировались функциональные возможности ФМ-подхода с теоретических позиций, разрабатывались критерии для обнаружения и распознавания нейроваскулярных структур, проводились экспериментальные исследования in vivo и in situ.

\section{Функциональные возможности фазово-модуляционного подхода}

Процесс распространения и ослабления излучения в биологических тканях in vivo и in situ определяется их оптическими свойствами, которые зависят от размера, концентрации и оптических свойств структурных элементов, таких как клетки, органеллы, биологические макромолекулы и другие внутриклеточные компоненты, от времени стабильности микроструктуры, величины исследуемого объема и протекающих физиологических процессов - в первую очередь гемодинамических, а также метаболических, электрофизиологических и патологических; последние сопровождаются изменениями состава и структуры на клеточном, внутриклеточном и тканевом уровнях $[31,32]$.

Для биологических тканей, кроме цельной крови, применимо диффузионное приближение нестационарной теории переноса излучения, одним из основных условий применимости которого является доминирование эффектов рассеяния излучения в среде над эффектами поглощения, что характерно для диапазона длин волн 650-900 nm, в котором возможно глубинное зондирование биологических тканей со слабой пигментацией [31, глава 7]. Диффузионное приближение позволяет получить аналитическое выражение для количественной интегральной оценки оптических параметров исследуемого объема среды, биологической ткани, таких как коэффициент поглощения $\left(\mu_{a}\right)$ и транспортный коэффициент рассеяния $\left(\mu_{s}^{\prime}\right)[31,36,37]$ и, следовательно, для решения обратных диагностических задач. Значения $\mu_{a}$ и $\mu_{s}^{\prime}$ должны соответствовать условию $\mu_{a} \ll \mu_{s}^{\prime}$, где $\mu_{s}^{\prime}=\mu_{s}(1-g), g-$ фактор анизотропии.

Для реализации разрабатываемого метода обнаружения и распознавания нейроваскулярных структур ис- ходными параметрами являются $\mu_{a}$ и $\mu_{s}^{\prime}$ зондируемого объема ткани без рассмотрения алгоритмов их вычисления по измеряемым оптическим параметрам. Для ФМ-подхода непосредственно измеряемыми на разных длинах волн и при разных расстояниях „источникприемник“ параметрами являются средний уровень интенсивности модулированного излучения, обратно рассеянного в биологической ткани, амплитуда модуляции этого излучения и сдвиг фазы по сравнению с исходным сигналом. Зондирующее излучение модулируется по интенсивности частотой в диапазоне от $50 \mathrm{MHz}$ до $1 \mathrm{GHz}$, при этом оптимальные значения отношения сигнал/шум соответствуют частотам модуляции порядка $110 \mathrm{MHz}$.

Известно, что по значениям $\mu_{a}$, полученным для разных длин волн зондирующего излучения, можно рассчитать концентрации основных хромофоров, вносящих наибольший вклад в поглощение излучения применяемых длин волн. Величина $\mu_{s}^{\prime}$ определяется структурными компонентами биологической ткани, их морфологическими особенностями, объемными концентрациями и соотношениями оптических параметров.

Основные и производные параметры спектрофотометрии с временным разрешением (TRS) и определяющие соотношения представлены в табл. 1. На основании указанных параметров возможна оценка компонентного состава, структуры и функциональной активности биотканей для их дифференциального анализа, для обнаружения и распознавания неоднородных включений, в том числе нервных стволов и крупных кровеносных сосудов.

Достоинствами ФМС, соответствующими основным требованиям, являются следующие: глубинное зондирование, измерение абсолютных значений оптических параметров исследуемого объема биологической ткани, высокая чувствительность метода к компонентному составу и структурным особенностям ткани, высокое временное разрешение метода, оптоволоконный подвод и регистрация излучения в режиме обратного рассеяния и возможность эндоскопической реализации, малые мощности и интенсивности излучения, отсутствие дополнительного риска от использования в ходе нейрохирургической операции, возможность многократного применения, оценка абсолютных значений физиологических параметров и их динамики, относительная экономичность технической реализации метода.

\section{Критерии обнаружения и распознавания нейроваскулярных структур}

По оптическим свойствам биологических тканей, в том числе по значениям $\mu_{a}$ и $\mu_{s}^{\prime}$ для разных тканей, международным научным сообществом накоплено огромное количество экспериментальных данных $[38,39]$. Однако эти данные получены при разных условиях и иногда отличаются в разы для одинаковых тканей и длин волн, что затрудняет их применение для моделирования, 
Таблица 1. Основные спектрофотометрические и физиологические параметры и определяющие соотношения

\begin{tabular}{|c|c|}
\hline Параметр & Обозначение \\
\hline $\begin{array}{c}\text { Абсолютные значения } \\
\text { коэффициента поглощения } \\
\text { и транспортного } \\
\text { коэффициента рассеяния }\end{array}$ & $\begin{array}{l}\mu_{a}, 1 / \mathrm{cm} \\
\mu_{s}^{\prime}, 1 / \mathrm{cm}\end{array}$ \\
\hline $\begin{array}{c}\text { Тканевая сатурация - } \\
\text { степень насыщения кислородом } \\
\text { гемоглобина в сосудах } \\
\text { исследуемого объёма ткани }\end{array}$ & $S_{t} \mathrm{O}_{2}, \%$ \\
\hline $\begin{array}{c}\text { Отношение концентрации } \\
\text { оксигенированного } \\
\text { гемоглобина к концентрации } \\
\text { дезоксигенированного гемоглобина } \\
\text { в единице исследуемого объёма ткани }\end{array}$ & $h=\frac{\left[\mathrm{HbO}_{2}\right]}{[\mathrm{HHb}]}$, rel. un. \\
\hline $\begin{array}{c}\text { Отношение коэффициента поглощения } \\
\text { в ближнем ИК диапазоне длин волн } \\
\text { к коэффициенту поглощения } \\
\text { в красной области длин волн }\end{array}$ & $U=\frac{\mu_{a}\left(\lambda_{2}\right)}{\mu_{a}\left(\lambda_{1}\right)}$, rel. un. \\
\hline $\begin{array}{c}\text { Концентрация общего гемоглобина } \\
\text { в исследуемом объёме ткани }\end{array}$ & {$[\mathrm{tHb}], \mu \mathrm{M}$} \\
\hline $\begin{array}{c}\text { Абсолютное и относительное } \\
\text { изменение коэффициентов } \\
\text { поглощения и } \\
\text { транспортного рассеяния } \\
\text { с увеличением длины волны } \\
\text { зондирующего излучения }\end{array}$ & $\begin{array}{c}\Delta \mu_{a}, 1 / \mathrm{cm} \\
D_{a}, \mathrm{~cm}^{-1} / \mu \mathrm{m} \\
\Delta \mu_{s}, 1 / \mathrm{cm} \\
D_{s}, \mathrm{~cm}^{-1} / \mu \mathrm{m}\end{array}$ \\
\hline
\end{tabular}

Определяющие соотношения и пояснения
$\mu_{a}=\lambda_{1}=\mu_{a}\left(\lambda_{1}\right)_{m}-\mu_{a}\left(\lambda_{1}\right)_{w}=\varepsilon_{\mathrm{HHb}}\left(\lambda_{1}\right) \cdot[\mathrm{HHb}]+\varepsilon_{\mathrm{HbO}_{2}}\left(\lambda_{1}\right) \cdot\left[\mathrm{HbO}_{2}\right] ;$ $\mu_{a}\left(\lambda_{2}\right)=\mu_{a}\left(\lambda_{2}\right)_{m}-\mu_{a}\left(\lambda_{2}\right)_{w}=\varepsilon_{\mathrm{HHb}}\left(\lambda_{2}\right) \cdot[\mathrm{HHb}]+\varepsilon_{\mathrm{HbO}_{2}}\left(\lambda_{2}\right) \cdot\left[\mathrm{HbO}_{2}\right]$ $\mu_{a}\left(\lambda_{1}\right)$ и $\mu_{a}\left(\lambda_{2}\right)$ - коэффициенты поглощения за счёт $\mathrm{HbO}_{2}$ и $\mathrm{HHb}$; $\varepsilon_{\mathrm{HHb}}\left(\lambda_{1}\right), \varepsilon_{\mathrm{HbO}_{2}}\left(\lambda_{1}\right), \varepsilon_{\mathrm{HHb}}\left(\lambda_{2}\right), \varepsilon_{\mathrm{HbO}_{2}}\left(\lambda_{2}\right)-$ молярные

коэффициенты экстинкции $\mathrm{HHb}$ и $\mathrm{HbO}_{2}$, соответственно на длинах волн $\lambda_{1}$ и $\lambda_{2} ;[\varepsilon]=\mu \mathrm{M}^{-1} * \mathrm{~cm}^{-1}$; $\mu_{a}\left(\lambda_{1}\right)_{m}$ и $\mu_{a}\left(\lambda_{2}\right)_{m}$ - коэффициенты поглощения, вычисляемые по измеряемым параметрам обратно рассеянного в ткани излучения; $\mu_{a}\left(\lambda_{1}\right)_{w}$ и $\mu_{a}\left(\lambda_{2}\right)_{w}$ - коэффициенты поглощения воды на длинах волн $\lambda_{1}$ и $\lambda_{2}$

при известной или заданной её концентрации в единице объёма исследуемой ткани;

выражения для вычисления $\mu_{a m}$ и $\mu_{s m}^{\prime}$ представлены в [32,33]; $S_{t} \mathrm{O}_{2}=\frac{\left[\mathrm{HbO}_{2}\right] \cdot 100}{\left[\mathrm{HbO}_{2}\right]+[\mathrm{HHb}]}=\frac{h \cdot 100}{h+1} ; h=\frac{\mathrm{S}_{t} \mathrm{O}_{2}}{100-\mathrm{S}_{t} \mathrm{O}_{2}} ;$

$\left[\mathrm{HbO}_{2}\right]$ - концентрация оксигенированного гемоглобина в исследуемом объёме ткани, $\mu$ mole/litre $=\mu \mathrm{M}$;

$[\mathrm{HHb}]$ - концентрация дезоксигенированного гемоглобина, $\mu \mathrm{M}$;

$\lambda_{2}>\lambda_{1}, \lambda_{1}-$ левее изобестической точки молярных коэффициентов экстинкции $\mathrm{HbO}_{2}$ и $\mathrm{HHb}(806 \mathrm{Hм})$, $\lambda_{2}$ - правее изобестической точки; $U=\frac{\mu_{a}\left(\lambda_{2}\right)}{\mu_{a}\left(\lambda_{1}\right)}=\frac{\varepsilon_{\mathrm{HHb}}\left(\lambda_{2}\right)+\varepsilon_{\mathrm{HbO}_{2}}\left(\lambda_{2}\right) \cdot\left[\mathrm{HbO}_{2}\right] /[\mathrm{HHb}]}{\varepsilon_{\mathrm{HHb}}\left(\lambda_{1}\right)+\varepsilon_{\mathrm{HbO}_{2}}\left(\lambda_{1}\right) \cdot\left[\mathrm{HbO}_{2} /[\mathrm{HHb}]\right]} ;$ $U=\frac{\varepsilon_{\mathrm{HHb}}\left(\lambda_{2}\right)+\varepsilon_{\mathrm{HbO}_{2}}\left(\lambda_{2}\right) \cdot \mathrm{S}_{t} \mathrm{O}_{2} /\left(100-\mathrm{S}_{t} \mathrm{O}_{2}\right)}{\varepsilon_{\mathrm{HHb}}\left(\lambda_{1}\right)+\varepsilon_{\mathrm{HbO}_{2}}\left(\lambda_{1}\right) \cdot \mathrm{S}_{t} \mathrm{O}_{2} /\left(100-\mathrm{S}_{t} \mathrm{O}_{2}\right)}$ $U=\frac{\varepsilon_{\mathrm{HHb}}\left(\lambda_{2}\right)+\varepsilon_{\mathrm{HbO}_{2}}\left(\lambda_{2}\right) \cdot h}{\varepsilon_{\mathrm{HHb}}\left(\lambda_{1}\right)+\varepsilon_{\mathrm{HbO}_{2}}\left(\lambda_{1}\right) \cdot h} ;$ $h=\frac{\varepsilon_{\mathrm{HHb}}\left(\lambda_{2}\right)-U \cdot \varepsilon_{\mathrm{HHb}}\left(\lambda_{1}\right)}{U \cdot \varepsilon_{\mathrm{HbO}}\left(\lambda_{1}\right)-\varepsilon_{\mathrm{HbO}_{2}}\left(\lambda_{2}\right)}$

$$
S_{t} \mathrm{O}_{2}=\frac{100 \cdot\left[\mu_{a}\left(\lambda_{1}\right) \cdot \varepsilon_{\mathrm{HHb}}\left(\lambda_{2}\right)-\mu_{a}\left(\lambda_{2}\right) \cdot \varepsilon_{\mathrm{HHb}}\left(\lambda_{1}\right)\right]}{\mu_{a}\left(\lambda_{1}\right) \cdot\left[\varepsilon_{\mathrm{HHb}}\left(\lambda_{2}\right)-\varepsilon_{\mathrm{HbO}_{2}}\left(\lambda_{2}\right)\right]-\mu_{a}\left(\lambda_{2}\right) \cdot\left[\varepsilon_{\mathrm{HHb}}\left(\lambda_{1}\right)-\varepsilon_{\mathrm{HbO}_{2}}\left(\lambda_{1}\right)\right]} ;
$$

$[\mathrm{tHb}]=\left[\mathrm{HbO}_{2}\right]+[\mathrm{HHb}] ; \Delta \mu_{a}=\mu_{a}\left(\lambda_{2}\right)-\mu_{a}\left(\lambda_{1}\right) ;$

$$
D_{a}=\frac{\Delta \mu_{a}}{\Delta \lambda}=\frac{\mu_{a}\left(\lambda_{2}\right)-\mu_{a}\left(\lambda_{1}\right)}{\lambda_{2}-\lambda_{1}} \text {; при } \lambda_{2}>\lambda_{1} \text {, }
$$

$\lambda_{1}$ - левее изобестической точки молярных коэффициентов экстинкции $\mathrm{HbO}_{2}$ и $\mathrm{HHb}(806 \mathrm{HM})$, $\lambda_{2}$ - правее изобестической точки; $\Delta \mu_{s}^{\prime}=\mu_{s}^{\prime}\left(\lambda_{2}\right)-\mu_{s}^{\prime}\left(\lambda_{1}\right)$; $D_{s}=\frac{\Delta \mu_{s}^{\prime}}{\Delta \lambda} ; D_{s}=\frac{\Delta \mu_{s}^{\prime}}{\Delta \lambda}=\frac{\mu_{s}^{\prime}\left(\lambda_{2}\right)-\mu_{s}^{\prime}\left(\lambda_{1}\right)}{\lambda_{2}-\lambda_{1}} ; \lambda_{2}>\lambda_{1}$

проведения количественных оценок и решения диагностических задач.

Указанные в табл. 1 спектрофотометрические параметры: $\mu_{a}(\lambda)$ и $\mu_{s}^{\prime}(\lambda),\left[\mathrm{HbO}_{2}\right],[\mathrm{HHb}],[\mathrm{tHb}], S_{t} \mathrm{O}_{2}, D_{a}$ и $D_{s}$ характеризуют локальную гемодинамику, обмен кислорода, структурные особенности исследуемого объема ткани и значительно отличаются для обескровленной ткани, объема ткани с микроциркуляторной гемодинамикой, с крупным артериальным сосудом, с венозным сосудом или нервным стволом/нервом (рис. 1). Исходя из указанного, в данной работе наличие крупного сосуда (рис. $1, b)$ или нерва (рис. $1, d$ ) анализируется на основе известных параметров соответственно цельной крови или нерва относительно интегральных оптических параметров $\mu_{a}(\lambda)_{0}$ и $\mu_{s}^{\prime}(\lambda)_{0}$ типового объема зондируемой ткани без крупных нейроваскулярных включений (рис. 1, $a, c$ ). Типовой объем исследуемой ткани, например опухолевой, имеет определенную структурную организацию на клеточном уровне и характеризуется определенным строением микроциркуляторного русла с соответствующими уровнями кровенаполнения и кровотока и значением концентрации общего гемоглобина в единице типового объема ткани $[\mathrm{tHb}]_{0}$.

Полученное при локальных измерениях значение концентрации общего гемоглобина $[\mathrm{tHb}]=\left[\mathrm{HbO}_{2}\right]+[\mathrm{HHb}]$ (в микромоль на литр ткани, $\mu \mathrm{M}$ ) (табл. 1) сравнивается с граничным значением $[\mathrm{tHb}]_{0}(\mu \mathrm{M})$ для типового объема исследуемой ткани, которое оценивается по известным зависимостям [31, стр. 468] через параметр кровенаполнения либо объемный кровоток, соответствующие физиологической норме, или с учетом увеличенной в $k_{1}$ раз перфузии опухоли, что выявляется 

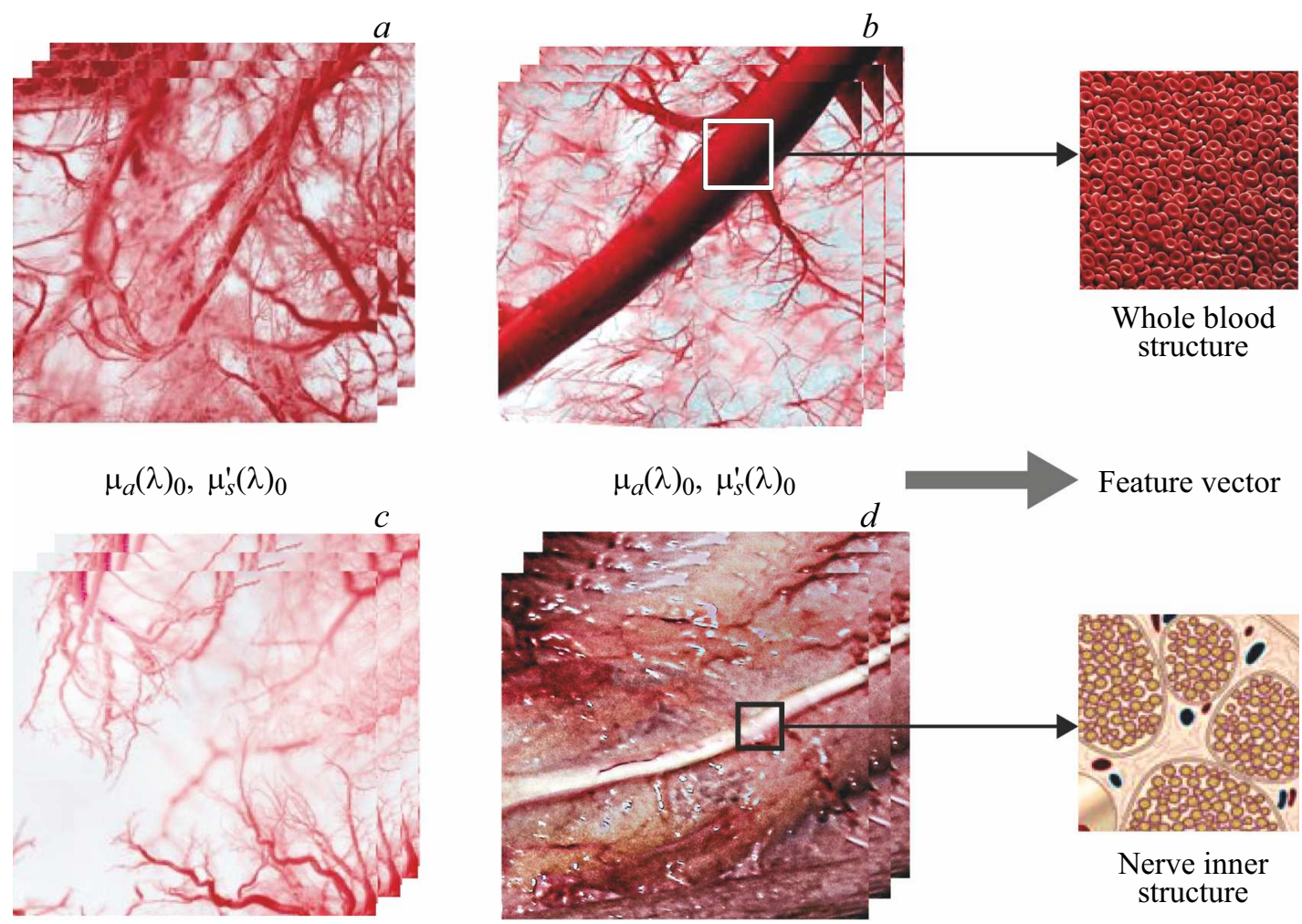

Рис. 1. Схематичное представление зондируемого объема биологической ткани: $(a)$ с высокой степенью васкуляризации на микроциркуляторном уровне, $(b)$ с крупным кровеносным сосудом артериальным или венозным, $(c)$ со сниженной васкуляризацией на микроциркуляторном уровне, $(d)$ с нервным стволом.

на этапе предоперационной диагностики, например, с помощью рентгеновской КТ. Учитываются индивидуальные параметры общего анализа крови, в частности, концентрация гемоглобина в единице объема крови, а также условия проведения операции, например, уменьшение объемного кровотока через коэффициент $k_{2}$ по отношению к предоперационному. Расчетная формула с учетом поправочных коэффициентов имеет вид

$$
[\mathrm{tHb}]_{0}=B V k_{1} k_{2} \frac{\rho_{t} C_{\mathrm{Hb}}}{M_{\mathrm{Hb}}} 10^{5},
$$

где $B V$ - объемное кровенаполнение биологической ткани (в миллилитрах крови на 100 г ткани , $\mathrm{mL} / 100 \mathrm{~g}$ ), соответствующее физиологической норме тканей головного мозга; $B V k_{1} k_{2}$ - объемное кровенаполнение опухолевой ткани $(\mathrm{mL} / 100 \mathrm{~g})$, с учетом увеличенной в $k_{1}$ раз перфузии опухоли и сниженного в $k_{2}$ раз минутного объема крови в условиях операции по отношению к предоперационному; $\rho_{t}$ - плотность исследуемой ткани $(1.03-1.05 \mathrm{~g} / \mathrm{mL}) ; C_{\mathrm{Hb}}-$ концентрация гемоглобина на $100 \mathrm{~mL}$ крови $(\mathrm{g} / 100 \mathrm{~mL}) ; M_{\mathrm{Hb}}$ - молярная масса гемоглобина $(64500 \mathrm{~g} / \mathrm{mole})$. Например, для тканей головного мозга вычисленные значения $[\mathrm{tHb}]_{0}$ при известных физиологических значениях $B V$ в норме для белого $(B V=0.3-0.5 \mathrm{~mL} / 100 \mathrm{~g})$ и серого $(B V=1-2 \mathrm{~mL} / 100 \mathrm{~g})$ вещества соответственно равны $8-12$ и $24-48 \mu \mathrm{M}$.
Если измеренное значение $[\mathrm{tHb}]$ в зондируемом объеме ткани превышает $[\mathrm{tHb}]_{0}$, то рассматривается либо случай увеличенной васкуляризации исследуемого объема на уровне микроциркуляторных сосудов, либо увеличение объема крови в исследуемом участке за счет наличия крупного кровеносного сосуда (рис. 1,b), либо его части, в зависимости от размеров сосуда по отношению к размерам зондируемого объема, глубины залегания и ориентации сосуда. Если измеренное значение $[\mathrm{tHb}]$ ниже $[\mathrm{tHb}]_{0}$, то рассматриваются случаи со сниженной перфузией на уровне микроциркуляции, либо с наличием другого типа ткани в исследуемом объеме, например, черепного нерва (рис. $1, d)$.

Для большинства мягких биологических тканей (мышечной, тканей мозга и др.) значения фактора анизотропии находятся в диапазоне от 0.7 до 0.95. Наличие крупного кровеносного сосуда как артериального, так и венозного в исследуемом объеме ткани, когда практически весь зондируемый объем занимает цельная кровь, идентифицируется по „аномальным“ изменениям $\mu_{s}^{\prime}, \mu_{s}^{\prime}\left(\lambda_{2}\right)>\mu_{s}^{\prime}\left(\lambda_{1}\right)$ при $\lambda_{2}>\lambda_{1}$, что является следствием нарушения основного условия применимости диффузионного приближения теории нестационарного переноса излучения, $\mu_{a} \ll \mu_{s}^{\prime}-\mu_{s}(1-g)$, так как для цельной крови $g=0.997-0.999$ [31, глава 3, стр. 223-229]. Учитывая существенные различия в значениях $g$ для крови 
и мягких тканей, обоснованно было бы предполагать, что при наличии крупного магистрального сосуда в зондируемом объеме ткани измеряемые значения $\mu_{s}^{\prime}(\lambda)$ будут существенно отличаться от значений $\mu_{s}^{\prime}(\lambda)_{0}$ для типового объема. Основной критерий обнаружения крупного кровеносного сосуда формулируется как $\Delta \mu_{s}^{\prime}>0$ или $D_{s}>0$.

Значения $\mu_{a}(\lambda)$ для биологических тканей в наибольшей степени зависят от величины кровенаполнения ткани [38]. Наличие крупного кровеносного сосуда изменит регистрируемое значение $\mu_{a}(\lambda)$ в сторону увеличения по сравнению с $\mu_{a}(\lambda)_{0}$ для типового объема исследуемой ткани.

Значения $S_{t} \mathrm{O}_{2} \geq 80 \%$ соответствуют повышенному содержанию артериальной крови. Ввиду того, что молярные коэффициенты экстинкции $\mathrm{HbO}_{2}$ и $\mathrm{HHb}$ существенно различаются для применимых в NIRS длин волн, кроме изобестической точки $806 \mathrm{~nm}$, различные „комбинации“ $\left[\mathrm{HbO}_{2}\right]$ и $[\mathrm{HHb}]$ в артериальной и венозной крови будут соответствовать разным значениям $\Delta \mu_{a}$ и $D_{a}$. Для артериальной крови с высоким уровнем насыщения гемоглобина кислородом характерны наибольшие значения $\left[\mathrm{HbO}_{2}\right], D_{a}>0$ и $\Delta \mu_{a}>0$, что следует из анализа данных по оптическим свойствам крови [31,глава 9] и соотношений табл. 1. При низком уровне тканевой сатурации значения $\Delta \mu_{a}<0$ и $D_{a}<0$.

Аналогичным образом, исходя из данных [38], наличие нервного ствола будет характеризоваться существенно меньшими значениями $\mu_{a}(\lambda)$ для исследуемого объема по сравнению с $\mu_{a}(\lambda)_{0}$, меньшими значениями $[\mathrm{tHb}]$ и $S_{t} \mathrm{O}_{2}$ по сравнению с аналогичными значениями типового объема ткани. Изменения $\mu_{s}^{\prime}(\lambda)$ с увеличением длины волны определяются степенным фактором $b$, так называемым „scattering power“ [38]. Различающиеся по структуре ткани и включения, кровь, липиды, нормальные ткани и неоплазии характеризуются разными значениями параметра $b$, определяющего разные скорости убывания $\mu_{s}^{\prime}(\lambda)$. Характерное строение и молекулярный состав нерва (с высоким содержанием липидов) определяют низкие значения $\mu_{a}(\lambda), \Delta \mu_{a}, D_{a},[\mathrm{tHb}]$ и $S_{t} \mathrm{O}_{2}$ для исследуемого объема ткани. Параметр $D_{s}$ принимает наименьшие значения по сравнению с величиной $D_{s} 0$ для выделенного элементарного объема исследуемой ткани, кроме жировой ткани, для которой величина $D_{s}$ также минимальна. Возможно, в случае липомы обнаружение нерва по параметру $D_{s}$ будет затруднено, что требует проверки.

Критерии обнаружения и распознавания нейроваскулярных структур должны быть независимыми параметрами, чувствительными к наличию крупного кровеносного сосуда или нерва в измеряемом объеме ткани. Предварительный анализ литературных данных позволяет предположить, что для обнаружения и распознавания крупных кровеносных сосудов целесообразно использовать вектор независимых параметров $\left(U, D_{a}\right.$, $\left.D_{s}\right)$, либо тождественный вектор $\left(S_{t} \mathrm{O}_{2},[\mathrm{tHb}], D_{s}\right)$, так как параметр $S_{t} \mathrm{O}_{2}$ связан взаимно однозначным соотношением с параметром $U$, и параметр $D_{a}$ функционально связан с $S_{t} \mathrm{O}_{2}$ и $[\mathrm{tHb}]$. Второй вариант вектора признаков отражает физиологические параметры и их динамику, доступнее для анализа и интерпретации медицинскими специалистами при отображении этих параметров в процессе нейрохирургической операции. Для автоматизированного обнаружения и распознавания предпочтительнее первый из предложенных векторов признаков, так как не требуется дополнительных расчетов физиологических параметров. Для распознавания нервов, предположительно, определяющими являются значения $\mu_{a}(\lambda)$ и $D_{s}$, отличающиеся от значений типового объема ткани и объема с крупным сосудом. Любой из двух предложенных выше вариантов вектора признаков может использоваться для распознавания нервов.

\section{Предварительные экспериментальные исследования}

\section{Материалы и оборудование}

Для проверки применимости предлагаемого вектора признаков и критериев исследовались in vivo области с различной анатомией сосудистого русла на предплечье, запястье и ладони у четырех добровольцев (женщин 20 лет (n1, n3, n4) и 47 лет (n2)) с целью обнаружения и распознавания крупных венозных и артериальных сосудов при наличии таковых в исследуемом объеме ткани (рис. 2). Зондировались следующие области: A - область предполагаемой локализации артерии (лучевой или локтевой), $\mathrm{V}$ - область локализации крупного венозного сосуда (подкожной вены), М область на предплечье, предположительно, не содержащая крупных кровеносных сосудов, Р и $\mathrm{F}$ - области соответственно на ладони и на подушечке пальца с высоким уровнем микроваскуляризации (рис. 2,a,b). Postmortum in situ исследовался один икроножный нерв (рис. 2,c) в шести зондируемых областях типа $\mathrm{N}$, предположительно, содержащих нерв, вдоль направления анатомического расположения нерва при нарушении верхних покровов тела. В двух случаях (\#3 и \#5) нерв попадал в зондируемый объем $\mathrm{N}$ (рис. 2,c), в остальных (\#1, 2, 4 и 6) зондирование проводилось через окружающие нерв ткани, кожножировой и мышечный слои.

Измерения проводились с помощью спектрофотометрического прибора „OxiplexTS“ (ISS, Inc., USA) c ФМ подходом (FD NIRS), принцип действия которого подробно рассмотрен в [36,37], и технические характеристики представлены на сайте производителя [40]. Зондирование биоткани осуществляется двумя длинами волн 692 и $834 \mathrm{~nm}$ от лазерных диодов мощностью $1 \mathrm{~mW}$. Интенсивность излучения модулируется по амплитуде с частотой $110 \mathrm{MHz}$ и глубиной модуляции 0.5. Регистрируется обратно рассе- 

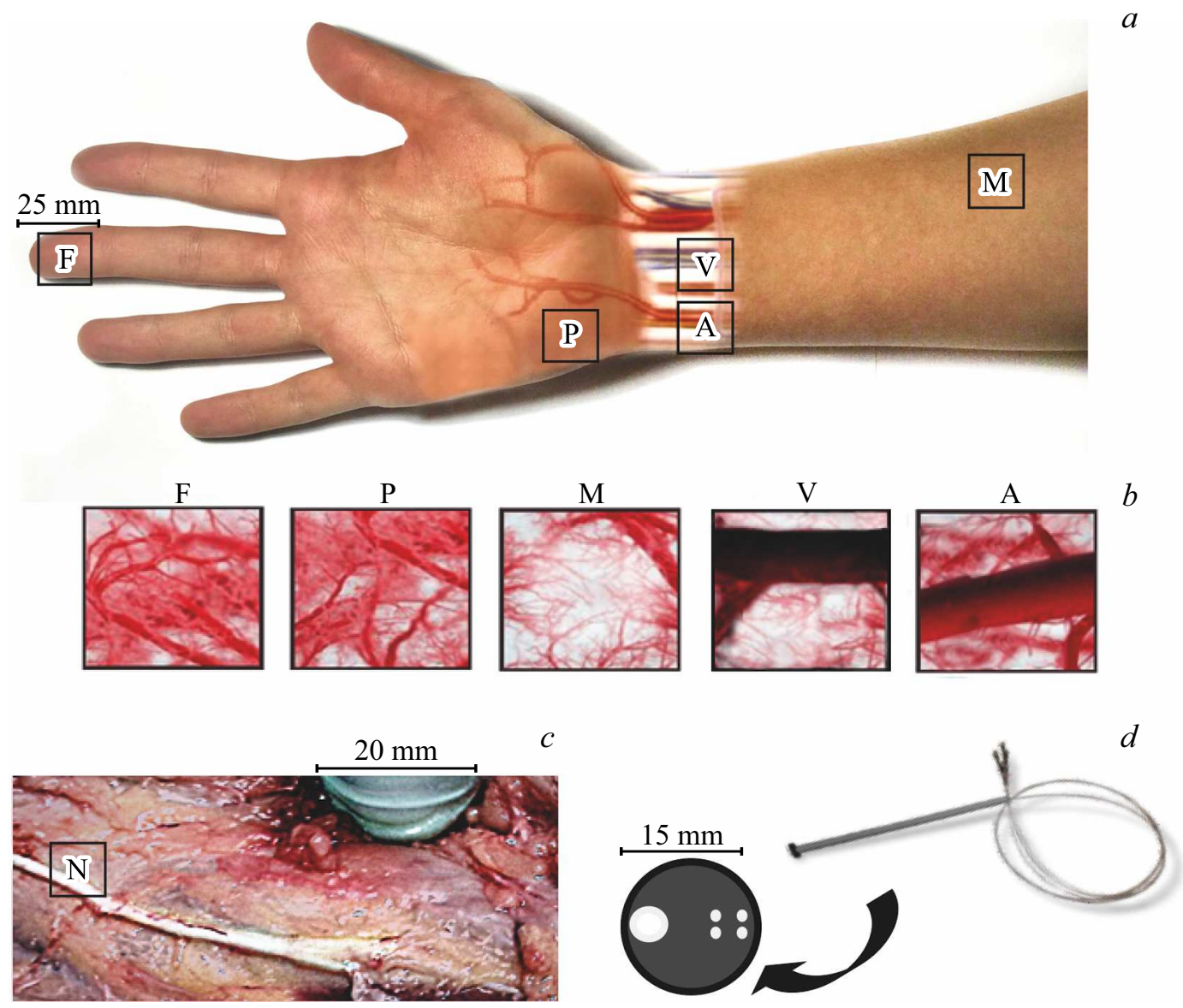

Рис. 2. Экспериментально исследуемые in vivo области на руке $(a)$ с различной структурой сосудистого русла $(b)$ без крупных сосудистых включений F, Р, М и с крупным артериальным А или венозным V сосудом; (c) исследуемая postmortum in situ область $\mathrm{N}$ икроножного нерва с помощью линейного оптоволоконного датчика $(d)$.

янное в ткани излучение с той же частотой модуляции. Калибровка прибора проводилась на калибровочном блоке с известными $\mu_{a}(\lambda)$ и $\mu_{s}^{\prime}(\lambda)$, близкими по оптическим свойствам к исследуемому типу биологической ткани, мышечной ткани при измерениях на руке in vivo и измерениях на ноге postmortum in situ.

Используемая для измерений рабочая модель оптоволоконного датчика-зонда характеризуется линейной конфигурацией (normal probe) с расстояниями между источниками и приемником 6 и $9 \mathrm{~mm}$ для каждой из длин волн (рис. 2, $d$ ). Излучение подводится по кварцполимерным волокнам (диаметр $400 \mu \mathrm{m}$ ), обратно рассеянное излучение передается на приемник по оптоволоконному жгуту диаметром $3 \mathrm{~mm}$. Примерная глубина зондирования $-2-5 \mathrm{~mm}$. Зондируемый объем биоткани составлял около $50-100 \mathrm{~mm}^{3}$.

Прибор „ОхірlexTS“ регистрирует абсолютные значения $\mu_{a}(692)$ и $\mu_{s}^{\prime}(692), \mu_{a}(834)$ и $\mu_{s}^{\prime}(834),\left[\mathrm{HbO}_{2}\right],[\mathrm{HHb}]$, $[\mathrm{tHb}]$ и $S_{t} \mathrm{O}_{2}$. Эти параметры интегрально косвенно оцениваются для зондируемого объема исследуемой биологической ткани по измеряемым амплитуде модуляции и сдвигу фаз по известным формулам [31,36,37].

\section{Экспериментальные результаты}

Результаты in vivo измерений по обнаружению и распознаванию сосудов предложенным методом показали существенные различия в значениях оптических и физиологических параметров (табл. 2) между областями регистрации $\mathrm{A}, \mathrm{V}, \mathrm{M}, \mathrm{P}$ и $\mathrm{F}$ для каждого испытуемого $(\mathrm{n} 1-\mathrm{n} 4)$, что соответствует отличиям в анатомическом строении и кровоснабжении разных исследуемых участков. Значения $\mu_{a}(692), \mu_{a}(834)$ и $[\mathrm{tHb}]$, соответствующие области М, в разы отличаются между испытуемыми (табл. 2), что определяется антропометрическими и возрастными различиями. Эти различия заключались в толщине подкожной жировой клетчатки и коррелировали с индексом массы тела (body mass index, BMI). Толщина подкожной жировой клетчатки и ВMI возрастали для испытуемых в следующем порядке $\mathrm{n} 2, \mathrm{n} 3, \mathrm{n} 1$, n4. В том же порядке убывали значения $\mu_{a}$ и [tHb]. Толщина кожножирового слоя в области М предплечья сопоставима с глубиной зондирования. Значение $[\mathrm{tHb}]$ из области М без крупных нейроваскулярных включений целесообразно принять за индивидуальное для каждого испытуемого значение $[\mathrm{tHb}]_{0}$, с которым сопоставляются 
Таблица 2. Результаты экспериментального исследования по обнаружению и распознаванию крупных кровеносных сосудов и нервов

\begin{tabular}{|c|c|c|c|c|c|c|c|c|c|c|c|}
\hline \multicolumn{12}{|c|}{ In vivo located arteries in A areas } \\
\hline n.\# & $\begin{array}{c}\mu_{a(692) m} \\
\mathrm{~cm}^{-1}\end{array}$ & $\begin{array}{c}\mu_{a(834) m} \\
\mathrm{~cm}^{-1}\end{array}$ & $\begin{array}{c}\mu_{a(692)} \\
\mathrm{cm}^{-1}\end{array}$ & $\begin{array}{l}\mu_{a(834)}, \\
\mathrm{cm}^{-1}\end{array}$ & $\begin{array}{c}\mu_{a(834)} / \\
\mu_{a(692)}\end{array}$ & $\begin{array}{c}\Delta \mu_{a} / \Delta \lambda, \\
\mathrm{cm}^{-1} / \mu \mathrm{m}\end{array}$ & $\begin{array}{l}\mu_{s(692)}^{\prime}, \\
\mathrm{cm}^{-1}\end{array}$ & $\begin{array}{l}\mu_{s(834)}^{\prime}, \\
\mathrm{cm}^{-1}\end{array}$ & $\begin{array}{l}\Delta \mu_{s}^{\prime} / \Delta \lambda, \\
\mathrm{cm}^{-1} / \mu \mathrm{m}\end{array}$ & $\begin{array}{c}S_{t} \mathrm{O}_{2} \\
\%\end{array}$ & $\begin{array}{c}{[\mathrm{tHb}],} \\
\mu \mathrm{M}\end{array}$ \\
\hline 1.1 & 0.231 & 0.267 & 0.227 & 0.242 & 1.066 & 0.106 & 15.1 & 11.6 & -24.6 & 72 & 109 \\
\hline 1.2 & 0.057 & 0.093 & 0.053 & 0.068 & 1.283 & 0.106 & 10.7 & 9.8 & -6.3 & 86 & 30 \\
\hline 2.1 & 0.165 & 0.279 & 0.161 & 0.254 & 1.578 & 0.655 & 11.5 & 12.7 & 8.4 & 88 & 110 \\
\hline 2.2 & 0.214 & 0.322 & 0.210 & 0.297 & 1.414 & 0.613 & 11.8 & 14.3 & 17.6 & 87 & 129 \\
\hline 2.3 & 0.100 & 0.200 & 0.096 & 0.175 & 1.823 & 0.556 & 8.4 & 13 & 32.4 & 93 & 73 \\
\hline 3.1 & 0.086 & 0.128 & 0.082 & 0.103 & 1.256 & 0.148 & 14.6 & 11.1 & -24.6 & 83 & 45 \\
\hline 4.1 & 0.048 & 0.082 & 0.044 & 0.057 & 1.295 & 0.092 & 6.4 & 6.0 & -2.8 & 89 & 25 \\
\hline \multicolumn{12}{|c|}{ In vivo located veins in $\mathrm{V}$ areas } \\
\hline 1.1 & 0.136 & 0.155 & 0.132 & 0.130 & 0.985 & -0.014 & 9.9 & 8.16 & -12.2 & 70 & 59 \\
\hline 1.2 & 0.2066 & 0.2784 & 0.203 & 0.253 & 1.246 & 0.352 & 12.6 & 16.1 & 24.6 & 80 & 113 \\
\hline 2.1 & 0.200 & 0.251 & 0.196 & 0.226 & 1.153 & 0.211 & 11.5 & 14.5 & 21.1 & 79 & 101 \\
\hline 2.2 & 0.235 & 0.230 & 0.231 & 0.205 & 0.887 & -0.183 & 8.7 & 9.2 & 3.8 & 63 & 95 \\
\hline 2.3 & 0.240 & 0.220 & 0.236 & 0.195 & 0.826 & -0.289 & 11.3 & 9.2 & -14.8 & 58 & 90 \\
\hline 3.1 & 0.1052 & 0.1137 & 0.101 & 0.088 & 0.871 & -0.092 & 8.4 & 7.9 & -3.5 & 66 & 41 \\
\hline \multicolumn{12}{|c|}{ In vivo located microcirculation in $\mathrm{M}$ areas } \\
\hline 1.1 & 0.079 & 0.1 & 0.0750 & 0.075 & 1.000 & -0.000 & 6.8 & 6.76 & -0.3 & 74 & 34 \\
\hline 2.1 & 0.165 & 0.16 & 0.1610 & 0.135 & 0.839 & -0.183 & 9.9 & 7.8 & -14.9 & 63 & 65 \\
\hline 2.2 & 0.118 & 0.141 & 0.1140 & 0.116 & 1.018 & 0.014 & 10.1 & 7.8 & -16.3 & 73 & 53 \\
\hline 2.3 & 0.140 & 0.150 & 0.1360 & 0.125 & 0.919 & -0.077 & 8.9 & 7.8 & -7.7 & 66 & 58 \\
\hline 3.1 & 0.103 & 0.135 & 0.0988 & 0.110 & 1.113 & 0.079 & 7.6 & 8.0 & 2.8 & 77 & 49 \\
\hline 4.1 & 0.067 & 0.081 & 0.0630 & 0.056 & 0.889 & -0.049 & 6.7 & 5.6 & -7.7 & 70 & 26 \\
\hline \multicolumn{12}{|c|}{ In vivo located microcirculation in $\mathrm{P}$ areas } \\
\hline 1.1 & 0.156 & 0.181 & 0.152 & 0.156 & 1.025 & 0.0267 & 20.9 & 16.3 & -32.4 & 73 & 70 \\
\hline 2.1 & 0.200 & 0.233 & 0.196 & 0.208 & 1.060 & 0.083 & 16.2 & 11.6 & -32.4 & 73 & 94 \\
\hline 2.2 & 0.213 & 0.254 & 0.209 & 0.229 & 1.095 & 0.139 & 16.3 & 13.3 & -21.174 & 103 & \\
\hline 2.3 & 0.180 & 0.181 & 0.176 & 0.156 & 0.885 & -0.142 & 16.5 & 13.0 & -24.6 & 64 & 70 \\
\hline \multicolumn{12}{|c|}{ In vivo located microcirculation in $\mathrm{F}$ areas } \\
\hline 1.1 & 0.156 & 0.222 & 0.152 & 0.197 & 1.295 & 0.315 & 21.9 & 16.3 & -39 & 82 & 87 \\
\hline \multicolumn{12}{|c|}{ Postmortum in situ located nerve } \\
\hline$\# 1$ & 0.193 & 0.152 & 0.189 & 0.127 & 0.673 & -0.435 & 7.4 & 6.7 & -5.1 & 57 & 63 \\
\hline$\# 2$ & 0.181 & 0.148 & 0.177 & 0.122 & 0.692 & -0.383 & 5.9 & 2.9 & -20.8 & 38 & 57 \\
\hline \#3 & 0.083 & 0.068 & 0.079 & 0.043 & 0.540 & -0.257 & 2.6 & 1.8 & -5.7 & 34 & 21 \\
\hline$\# 4$ & 0.187 & 0.124 & 0.183 & 0.099 & 0.540 & -0.594 & 7.0 & 4.1 & -20.1 & 37 & 52 \\
\hline \#5 & 0.067 & 0.061 & 0.063 & 0.036 & 0.568 & -0.191 & 2.2 & 1.6 & -4.1 & 28 & 15 \\
\hline \#6 & 0.237 & 0.145 & 0.2333 & 0.120 & 0.515 & -0.797 & 5.4 & 2.5 & -20.1 & 36 & 66 \\
\hline
\end{tabular}

значения $[\mathrm{tHb}]$, регистрируемые в областях А и $\mathrm{V}$ при обнаружении крупных сосудов.

Наименьшие отрицательные значения $D_{s}$, соответствующие резкому убыванию значений $\mu_{s}^{\prime}$ при увеличении длины волны от 692 до $834 \mathrm{~nm}$, относятся к областям $\mathrm{P}$ и $\mathrm{F}$ с максимальной васкуляризацией на микроциркуляторном уровне и минимальной толщиной слоя кожи (табл. 2). Последний результат согласуется с данными литературы [31, стр. 66]. Чем меньше размеры структурных элементов исследуемого объема, тем быстрее убывает $\mu_{s}^{\prime}$ с длиной волны. Максимальная васкуляризация и минимальное из всех регистрируемых значений $D_{s}$ соответствуют области $\mathrm{F}$ на подушечке пальца руки (табл. 2).

Существенная неоднородность данных для области А при обнаружении артерии объясняется отсутствием точных данных об индивидуальном анатомическом строении, глубиной залегания артерии при невозможности контролировать ее положение относительно поверхности кожи, неточностью позиционирования датчика и узостью зондирования в силу линейной геометрии датчика (рис. 2,d), плотным расположением структур с 
разными оптическими свойствами в области запястья, включая артерии, вены, нервы, костные, мышечные и соединительнотканные структуры. Неоднородность данных для области V при обнаружении вены объясняется тем, что для 20-летних испытуемых подкожные вены были практически не видны, а также неточностью позиционирования и особенностью геометрии датчика. У испытуемого n2 расположение подкожных вен легко определялось визуально.

Абсолютные значения $\mu_{a}(\lambda)$ и $[\mathrm{tHb}]$ в значительной мере зависят от состояния микроциркуляторного русла исследуемой биологической ткани, что также влияет на пороговые значения для $D_{a}$ и $[\mathrm{tHb}]$ предлагаемых векторов признаков для обнаружения и распознавания крупных сосудов. Регистрируемые значения параметров, например $[\mathrm{tHb}$, для исследуемого объема можно нормировать на величину $[\mathrm{tHb}]_{0}$ для типового объема. Тогда нормированные значения параметров для исследуемых областей будут больше или меньше 1, либо близки к 1 в случае соответствия свойств исследуемой области свойствам типового объема биоткани. В этом случае возможна выработка универсального критерия на основе безразмерного признака, инвариантного к свойствам окружающей сосуд ткани и чувствительного к объемному кровенаполнению. Максимальные физиологические значения $[\mathrm{tHb}]$ для пальцев рук при максимальном кровоснабжении соответствуют примерно $150-180 \mu \mathrm{M}$, в зависимости от конституции человека, объема циркулирующей крови и ее свойств, что нетрудно оценить, используя литературные данные по физиологии кровообращения.

Полученные экспериментальные данные (табл. 2) соответствуют априорной физиологической информации. При попадании крупных кровеносных сосудов, артериальных или венозных в исследуемый объем отношение $\Delta \mu_{s}^{\prime} / \lambda>0$. Для объема с мелкими сосудами микроциркуляторного уровня отношение $\Delta \mu_{s}^{\prime} / \Delta \lambda<0$. Крупные артериальные и венозные сосуды различаются по регистрируемому уровню $S_{t} \mathrm{O}_{2}-$ значения $S_{t} \mathrm{O}_{2} \geq 80 \%$ соответствуют артериям, $S_{t} \mathrm{O}_{2}<80 \%-$ венам (табл. 2 и рис. 3). Наличие артериоловенулярных шунтов в области F определяет высокие значения объема артериальной крови, концентрации оксигенированного гемоглобина и значения $S_{t} \mathrm{O}_{2} \geq 80 \%$. Для достоверного распознавания необходимы другие параметры предложенного вектора признаков и соответствующие критерии.

Параметр $S_{t} \mathrm{O}_{2}$ функционально связан с параметрами $h=\left[\mathrm{HbO}_{2}\right] /[\mathrm{HHb}]$ и $U=\mu_{a}\left(\lambda_{2}\right) / \mu_{a}\left(\lambda_{1}\right)$ по формулам, указанным в табл. 1, что представлено на рис. 3. Для параметра $U=\mu_{a}(834) / \mu_{a}(692)$ экспериментально полученные значения располагаются ниже теоретической кривой (рис. 3). Для параметра $h$ теоретические и экспериментальные результаты практически совпадают. Отклонения экспериментальных данных для $U$, возможно, объясняются методической и инструментальной погрешностями спектрофотометра „ОxiplexTS“, используемого для измерений, несовершенством алгоритма разделения эффек- тов поглощения и рассеяния, либо отражают реальные процессы распространения излучения в кровоснабжаемой биологической ткани. Известно, что цельная кровь сильнее ослабляет зондирующее излучение, чем гемолизированная, при одинаковых концентрациях фракций гемоглобина. Большей чувствительностью к изменениям тканевой сатурации в диапазоне от 80 до $100 \%$ обладает параметр $h$. По параметрам $S_{t} \mathrm{O}_{2}, U$ или $h$ области $\mathrm{M}, \mathrm{P}$ и $\mathrm{F}$, отличающиеся строением сосудистого русла и кровенаполнением, неразличимы. Значения $\mu_{s}^{\prime}(\lambda)$ и $D_{s}$ определяются и морфологическими особенностями тканей на клеточном уровне, и структурными тканевыми элементами, например строением сосудистого русла и объемным кровенаполнением. Чем выше структурированность и гетерогенность на тканевом уровне, тем больше значения $\mu_{s}^{\prime}(\lambda)$ и меньше значения $D_{s}$.

Результаты измерений по обнаружению и распознаванию нерва приведены в табл. 2. Результаты единичного эксперимента не противоречат сделанным выше предположениям относительно предлагаемых признаков и соответствующих критериев, однако требуют проверки и верификации на большом объеме экспериментальных данных. Низкие значения параметров $S_{t} \mathrm{O}_{2}, U$ или $h$, полученные при измерениях для икроножного нерва postmortum in situ, объясняются отсутствием кровообращения в области измерений. Живые биологические ткани в норме характеризуются значениями $S_{t} \mathrm{O}_{2}>55 \%$ и $\left[\mathrm{HbO}_{2}\right]>[\mathrm{HHb}]$ в условиях притока артериальной крови и поставки кислорода, адекватной метаболическим потребностям тканей.

Применение предлагаемых критериев и векторов признаков позволяет разделить зондируемые объемы на следующие группы: с преобладающей микроциркуляторной васкуляризацией (высокого и низкого уровня), с крупным артериальным сосудом, с крупным венозным сосудом и с нервом. Дополнительные независимые критерии для распознавания васкулярных структур в объеме ткани позволяет выработать анализ выраженности и характера пульсовых колебаний для разных участков сосудистого русла с учетом наличия ангиопатии по специально разрабатываемым алгоритмам. Анализ других составляющих медленных гемодинамических колебаний в исследуемом объеме ткани: дыхательной, миогенной, нейрогенной и эндотелиальной затруднен или невозможен в силу ограниченного времени проведения одномоментных измерений в условиях нейрохирургической операции.

\section{Обсуждение}

Основные ограничения ФМС связаны с контактностью измерений и зависимостью глубины зондирования от расстояния между источником и приемником. В первом случае неизбежны двигательные артефакты в измеряемом оптическом сигнале от изменения усилия прижатия датчика к поверхности биологической ткани, что 


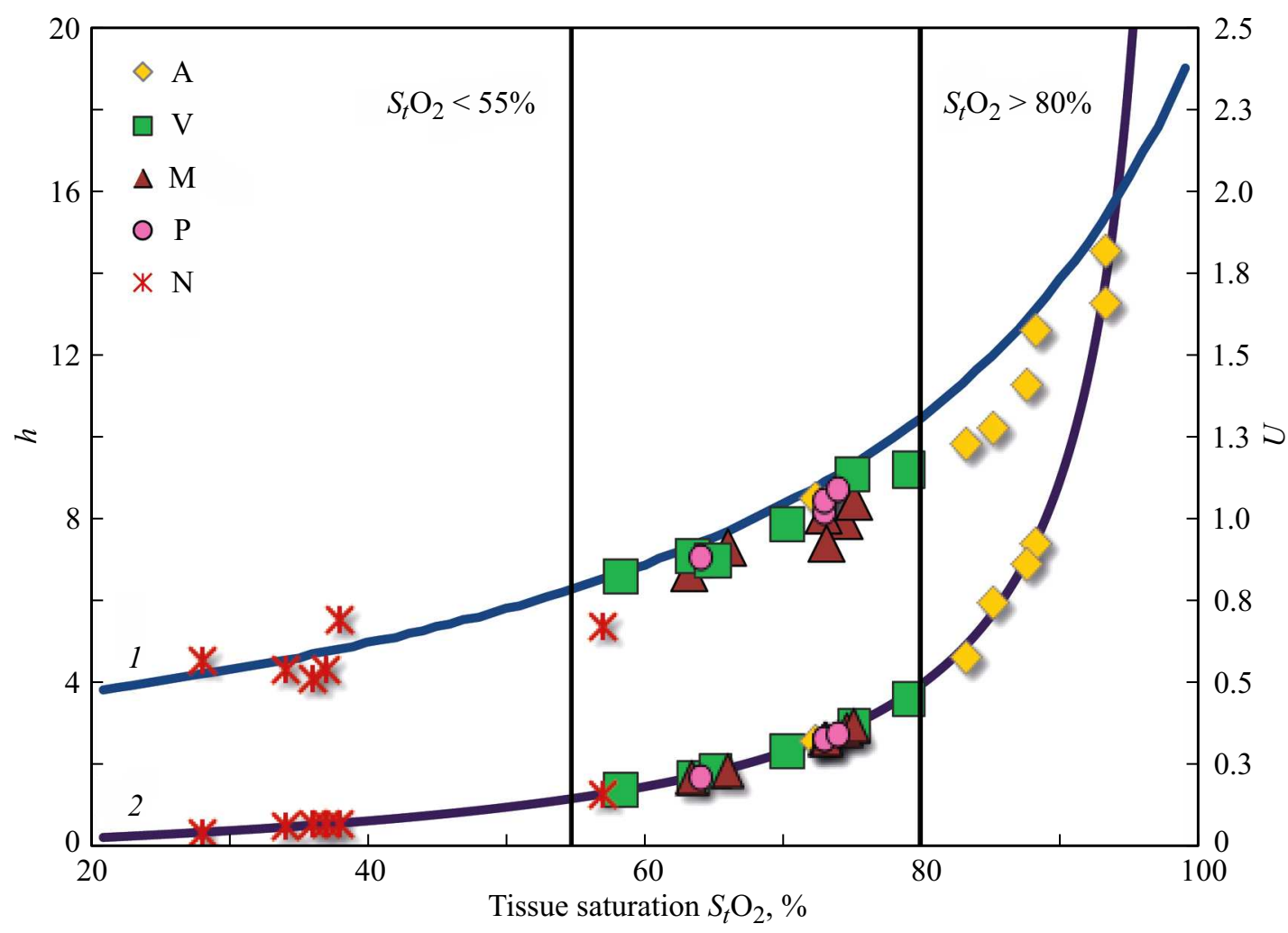

Рис. 3. Графики теоретических зависимостей между диагностическими параметрами $S_{t} \mathrm{O}_{2}, U$ и $h$ и результаты измерений для различных участков зондирования in vivo при обнаружении и распознавании крупных артериальных $(\mathrm{A})$ и венозных (V) сосудов и нервов $(\mathrm{N})$ и для областей $(\mathrm{M})$ и $(\mathrm{P})$ с микроциркуляторной гемодинамикой без крупных нейроваскулярных включений.

приводит к отклонениям контролируемых параметров, к возможным ошибкам в обнаружении и распознавании нейроваскулярных структур. Вязкоупругие свойства биологических тканей являются причиной существенного недостатка контактных измерений с неконтролируемым усилием прижатия. Кровенаполнение ткани, структура и, следовательно, количественные оценки зависят от усилия прижатия датчика к поверхности биоткани рукой хирурга и вариабельности этого усилия в течение времени измерения. Наилучшие по воспроизводимости результаты получаются при фиксации оптического датчиказонда контактно с поверхностью с помощью штатива без деформации тканей и сосудов, что выполнялось при экспериментальных исследованиях in vivo, но невозможно в реальных условиях проведения трансназальной нейрохирургической операции. Для решения данной проблемы применимы специальные методы анализа динамики регистрируемых параметров и алгоритмов обработки с компенсацией двигательных артефактов [41]. Достоинством контактных методов является возможность калибровки прибора и проведения измерений в условиях минимальной засветки, приемлемой для регистрации информативных сигналов. Идеальным решением является роботизированный нейрохирургический датчик-зонд с обратной связью по наличию контакта и контролю усилия прижатия для стандартизации измерений с учетом засветки и других условий применения. На текущий момент перспективным является бесконтактное зондирование, что требует глубокой проработки в силу нетривиальности решения.

Для нейрохирургии трансназального доступа метод ФМС должен быть реализован узкодиаметральным датчиком диаметром не более $6 \mathrm{~mm}$. Поскольку глубина зондирования напрямую зависит от расстояния между источником и приемником излучения, необходима специальная конструкция датчика с раздвижным окончанием, или с косым срезом торца, или специальным расположением осветителя вне плоскости приемника. Вариабельность конструкции позволит обеспечить глубину зондирования $2-5 \mathrm{~mm}$ и более. Планарное расположение источников и приемников на рабочем конце датчика обеспечит инвариантность метода регистрации к направлению расположения нейроваскулярной структуры в зондируемом объеме. Доступ к новообразованию и последующие манипуляции нейрохирурга осуществляются либо через один носовой канал, либо через оба канала одновременно. В первом случае узкодиаметральный спектрофотометрический датчик может вводиться совместно с эндоскопом и эндоскопическим нейрохирургическим инструментарием. Во втором случае датчик используется как отдельный инструмент, вводимый в свободный носовой канал, что дает возможность опционального использования датчика в процессе операции. Перспективным направлением для создания 
многофункциональных, технологичных и экономичных инструментов для нейрохирургии является разработка волноводов на основе кристаллов сапфира заданной формы [42].

Расширение диапазона длин волн в ближней инфракрасной области, применение длины волны $930 \mathrm{~nm}$, соответствующей локальному максимуму поглощения липидов [38] и длины волны $1000 \mathrm{~nm}$, где доминирует поглощение водой, позволят повысить эффективность распознавания нервных стволов. Для внедрения разрабатываемого метода и его корректировки с учетом специфики нейрохирургической операции необходимы данные по оптическим свойствам основных видов новообразований головного мозга, данные предоперационного исследования кровоснабжения опухоли и измерения интегральных оптических параметров опухоли перед резекцией непосредственно в процессе операции.

Для оценки чувствительности и специфичности разрабатываемого метода к размерам и глубине залегания различных нейроваскулярных структур необходимо проведение статистического имитационного моделирования, например, методом Монте-Карло. Для экспериментального подтверждения необходимо физическое моделирование на фантомах, имитирующих оптические свойства и различную топологию биологических тканей. По статическим фантомам биологических тканей для оптических измерений существует достаточный объем литературных данных [31]. Границы количественных критериев, зависящих от значений $\mu_{a}(\lambda)$ с учтенной поправкой на содержание воды, возможно, потребуют корректировки в зависимости от процентного содержания цельной крови крупного сосуда в исследуемом объеме. Например, при $70 \%$ содержании воды в ткани молярная концентрация воды составляет $38.9 \mathrm{M}$ (mole/litre). Цельной крови с показателем гематокрита $45 \%$ соответствует молярная концентрация воды около $30.6 \mathrm{M}$. Значимость различий будет зависеть от длин волн зондирующего излучения и достижимой чувствительности фазово-модуляционных измерений.

\section{Заключение}

Полученные предварительные экспериментальные результаты и их соответствие литературным данным по физиологии и оптическим свойствам биологических тканей подтверждают эффективность ФМС для обнаружения и распознавания нейроваскулярных структур в объеме биоткани, состоятельность выдвинутых предположений относительно критериев обнаружения и распознавания. Для окончательной верификации выдвинутых гипотез, оценки граничных значений предложенных критериев и выработки решающих правил автоматизированного обнаружения и распознавания нейроваскулярных структур в объеме ткани необходимо проведение исследований in situ в реальных условиях с помощью рабочей модели оптического датчика-зонда для трансназальной нейрохирургии, спроектированного с учетом всех медико-технических требований к нему. Открытыми остаются вопросы оценки точности, воспроизводимости, чувствительности и специфичности разрабатываемого метода на основе ФМС. Соответствующие оценки зависят от параметров зондирующего излучения, конструктивных особенностей технической реализации метода, включая геометрию датчика, обеспечивающую определенную глубину зондирования и пространственное разрешение с учетом оптических свойств исследуемых биологических тканей. Применительно к нейрохирургии актуальным является создание базы данных по оптическим свойствам опухолей тканей головы и головного мозга. Дальнейшее развитие метода возможно также с учетом современных тенденций в спектрофотометрии с временным разрешением (TRS), например, на базе временного подхода (TD). Полученные в процессе данного исследования результаты в равной мере применимы к TD NIRS. Разрабатываемый спектрофотометрический метод совместим с другими оптическими методами диагностики для повышения эффективности эндоскопической трансназальной нейрохирургии. Несомненным преимуществом обладают мультимодальные варианты решений, совмещающие нейронавигационные возможности, мультиспектральную визуализацию, глубинное зондирование и количественную дифференциальную оценку с высоким пространственным разрешением при минимальных габаритах датчика-зонда для решения задач эндоскопической нейрохирургии основания черепа.

\section{Соблюдение этических стандартов}

Все процедуры, выполненные в исследовании с участием людей, соответствуют этическим стандартам институционального и/или национального комитета по исследовательской этике и Хельсинкской декларации 1964 г. и ее последующим изменениям или сопоставимым нормам этики.

\section{Конфликт интересов}

Авторы заявляют, что у них нет конфликта интересов.

\section{Список литературы}

[1] Snyderman C.H., Carrau R.L., Kassa A.B., Zanation A., Prevedello D., Gardner P., Mintz A. // J. Surg. Oncol. 2008. V. 97. N 8. P. 658. doi 10.1002/JSO. 21020

[2] Shkarubo A.N., Koval K.V., Chernov I.V., Andreev D.N., Panteleyev A.A. // World Neurosurg. 2019. V. 121. P. 246. doi 10.1016/j.wneu.2018.09.090

[3] Shkarubo A.N., Chernov I.V., Ogurtsova A.A., Moshchev D.A., Lubnin A.J., Andreev D.N., Koval K.V. // World Neurosurg. 2017. V. 98. P. 230. doi 10.1016/J.WNEU.2016.10.089 
[4] Chan S., Conti F., Salisbury K., Blevins N.H. // Neurosurgery. 2013. V. 72. N 1. P. A154. doi 10.1227/NEU.0b013e3182750d26

[5] Cordero E., Latka I., Matth.us C., Schie I.W., Popp J. // J. Biomed. Opt. 2018. V. 23. N 7. P. 071210. doi 10.1117/1.JBO.23.7.071210

[6] Wisotzky E.L., Uecker F.C., Arens P., Dommerich S., Hilsmann A., Eisert P. // J. Biomed. Opt. 2018. V. 23. N 9. P. 091409. doi 10.1117/1.JBO.23.9.091409

[7] Gono K., Obi T., Yamaguchi M., Ohyama N., Machida H., Sano Y., Yoshida S., Hamamoto Y., Endo T. // J. Biomed. Opt. 2004. V. 9. N 3. P. 568. doi 10.1117/1.1695563

[8] Bolton F.J., Bernat A.S., Bar-Am K., Levitz D., Jacques S. // J. Biomed. Opt. 2018. V. 23. N 12. P. 121612. doi 10.1117/1.JBO.23.12.121612

[9] Baran U., Wang R.K. // Neurophotonics. 2016. V. 3. N 1. P. 010902. doi 10.1117/1.NPh.3.1.010902

[10] Assayag O., Grieve K., Devaux B., Harms F., Pallud J., Chretien F., Boccara C., Varlet P. // NeuroImage: Clinical. 2013. V. 2. N 1. P. 549. doi 10.1016/J.NICL.2013.04.005

[11] Bachmann L., Zezell D.M., Ribeiro A.C., Gomes L., Ito A.S. // Appl. Spectrosc. Rev. 2006. V. 41. N 6. P. 575. doi 10.1080/05704920600929498

[12] Sun Y., Hatami N., Yee M., Phipps J., Elson D.S., Gorin F., Schrot R.J., Marcu L. // J. Bi-omed. Opt. 2010. V. 15. N 5. P. 056022. doi 10.1117/1.3486612

[13] Pogue B.W., Gibbs-Strauss S.L., Valdes P.A., Samkoe K.S., Roberts D.W., Paulsen K.D. // IEEE J. Select. Top. Quant. Electron. 2010. V. 1. N 3. doi 10.1109/JSTQE.2009.2034541

[14] Oh S.J., Kim S.-H., Ji Y.B., Jeong K., Park Y., Yang J., Park D.W., Noh S.K., Kang S.-G., Huh Y.-M., Son J.-H., Suh J.-S. // Biomed. Opt. Express. 2014. V. 5. N 8. P. 2837. doi 10.1364/BOE.5.002837

[15] Gavdush A.A., Chernomyrdin N.V., Malakhov K.M., Beshplav S.-I.T., Dolganova I.N., Kosyrkova A.V., Nikitin P.V., Musina G.R., Katyba G.M., Reshetov I.V., Cherkasova O.P., Komandin G.A., Karasik V.E., Potapov A.A., Tuchin V.V., Zaytsev K.I. // J. Biomed. Opt. 2019. V. 24. N 2. 027001. doi 10.1117/1.JBO.24.2.027001

[16] Utzinger U., Richards-Kortum R.R. // J. Biomed. Opt. 2003. V. 8. N 1. P. 121. doi $10.1117 / 1.1528207$

[17] Осипов Л.В. Ультразвуковые диагностические приборы: режимы, методы и технологии. М.: ООО ПКФ .ИзоМед., 2011. $316 \mathrm{c}$

[18] Uribe J.S., Vale F.L., Dakwar E. // Spine. 2010. V. 35. N $26 \mathrm{~S}$. P. S368. doi 10.1097/BRS.0b013e3182027976

[19] Wells J., Konrad P., Kao C., Jansen E.D., MahadevanJansen A. // J. Neurosci. Meth. 2007. V. 163. N 2. P. 326. doi 10.1016/J.JNEUMETH.2007.03.016

[20] Wells J., Kao C., Konrad P., Milner T., Kim J., MahadevanJansen A., Jansen E.D. // Bio-phys. J. 2007. V. 93. N 7. P. 2567. doi 10.1529/BIOPHYSJ.107.104786

[21] Izzo A.D., Walsh J.T., Ralph H., Webb J., Bendett M., Wells J., Richter C.-P. // Biophys. J. 2008. V. 94. N 8. P. 3159. doi 10.1529/BIOPHYSJ.107.117150

[22] Cayce J.M., Friedman R.M., Jansen E.D., MahavadenJansen A., Roe A.W. // NeuroImage. 2011. V. 57. N 1. P. 155. doi 10.1016/J.NEUROIMAGE.2011.03.084

[23] Jeschke M., Moser T. // Hearing Research. 2015. V. 322. P. 224. doi 10.1016/J.HEARES.2015.01.005
[24] Teudt I.U., Nevel A.E., Izzo A.D., Walsh J.T., Richter C.-P. // Laryngoscope. 2007. V. 117. P. 1641. doi 10.1097/MLG.0b013E318074EC00

[25] Smistad E., Johansen K.F., Iversen D.H., Reinertsen I. // J. Med. Imag. 2018. V. 5. N 4. P. 044004. doi 10.1117/1.JMI.5.4.044004

[26] Miner R.C. // J. Med Imaging and Radiation Sci. 2017. V. 48. I. 4. P. 328. doi 10.1016/j.jmir.2017.06.005

[27] Kircher M.F., A. de la Zerda, Jokerst J.V., Zavaleta C.L., Kempen P.J., Mittra E., Pitter K., Huang R., Campos C., Habte F., Sinclair R., Brennan C.W., Mellinghoff I.K., Holland E.C., Gambhir S.S. // Nature Medicine. 2012. V. 18. N 5. P. 829. doi $10.1038 / \mathrm{nm} .2721$

[28] Wolf M., Ferrari M., Quaresima V. // J. Biomed. Opt. 2007. V. 12. N 6. P. 062104. doi 10.1117/1.2804899

[29] Ferrari M., Quaresima V. // NeuroImage. 2012. V. 63. N 2. P. 921. doi 10.1016/J.NEUROIMAGE.2012.03.049

[30] Yucel M.A., Selb J.J., Huppert T.J., Franceschini M.A., Boas D.A. // Curr. Opin. Biomed. Eng. 2017. V. 4. P. 78. doi 10.1016/J.COBME.2017.09.011

[31] Оптическая биомедицинская диагностика / Под ред. Тучина В.В. Т. 1. М.: Физматлит, 2007. 560 с. Handbook of Optical Biomedical Diagnostics/Ed. by Tuchin V.V. Bellingham: SPIE Press. 2002. 1110 p.

[32] Tuchin V.V. // J. Biomed. Photon. Eng. 2015. V. 1. N 2. P. 98. doi 10.18287/JBPE-2015-1-2-98

[33] Chitnis D., Airantzis D., Highton D., Williams R., Phan P., Giagka V., Powell S., Cooper R.J., Tachtsidis I., Smith M., Elwell C.E., Hebden J.C., Everdell N. // Rev. Sci. Instrum. 2016. V. 87. N 6. P. 065112 . doi $10.1063 / 1.4954722$

[34] Mazurenka M., Di Sieno L., Boso G., Contini D., Pifferi A., Mora A.D., Tosi A., Wabnitz H., Macdonald R. // Biomed. Opt. Express. 2013. V. 4. N 10. P. 2257. doi 10.1364/BOE.4.002257

[35] Pifferi A., Contini D., Mora A.D., Farina A., Spinelli L., Torricelli A. // J. Biomed. Opt. 2016. V. 21. N 9. P. 091310. doi 10.1117/1.JBO.21.9.091310

[36] Fantini S., Franceschini M.A., Maier J.S., Walker S.A., Barbieri B., Gratton E. // Optical Engin. 1995. V. 34. N 1. P. 32. doi $10.1117 / 12.183988$

[37] Fantini S., Franceschini M. A., and Gratton E. // J. Opt. Soc. Am .B. 1994. V. 11. N 10. P. 2128. doi 10.1364/JOSAB.11.002128

[38] Jacques S.L. // Phys. Med. Biol. 2016. V. 58. N 11. P. R37. doi 10.1088/0031-9155/58/11/R37

[39] Tuchin V.V. // J. Biomed. Photon. Eng. 2015. V. 1. N 1. P. 3. doi 10.18287/JBPE-2015-1-1-3

[40] Сайт компании ISS, Inc. Tissue oximeter „OxiplexTS“. [Электронный ресурс] Режим доступа: http://www.iss.com/biomedical/instruments/oxiplexTS.html.

[41] Brigadoi S., Ceccherini L., Cutini S., Scarpa F., Scatturin P., Selb J., Gagnon L., Boas D.A., Cooper R.J. // NeuroImage. 2014. V. 85. N 1. P. 181. doi 10.1016/j.neuroimage.2013.04.082

[42] Katyba G.M., Zaytsev K.I., Dolganova I.N., Shikunova I.A., Chernomyrdin N.V., Yurchenko S.O., Komandin G.A., Reshetov I.V., Nesvizhevsky V.V., Kurlov V.N. // Progress in Crystal Growth and Characterization of Materials. 2018. V. 64. I. 4. P. 133. doi 10.1016/j.pcrysgrow.2018.10.002 